\section{Mobilities of Envelope Proteins of Escherichia coli in Two-Dimen- sional SDS-polyacrylamide Gel Electrophoresis with Different Buffer Systems}

\author{
Koji Yoda and Gakuzo Tamura
}

\author{
Department of Agricultural Chemistry, University \\ of Tokyo, Bunkyo-ku, Tokyo 113
}

Received July 7, 1977

SDS-polyacrylamide gel electrophoresis (SDS/PAGE) played an important role in the analyses of envelope proteins of Escherichia coli as well as in those of other organisms. Because different protein profiles were obtained in many laboratories, ambiguity remains in identities of the proteins under biochemical characterizations (Identity of the major outer membrane proteins was discussed by Ichihara and Mizushima. ${ }^{1)}$ ) One of the origins of difference was attributed to conditions of solubilization. ${ }^{2)}$ Some of the major outer membrane proteins show different mobilities in SDS/ PAGE depending on the temperature of solubilization (heat-modifiability). ${ }^{3)}$ Some migrate upward and some migrate downward in SDS/PAGE after heating. $\mathrm{Na}$ kamura and Mizushima ${ }^{4)}$ considered this property of outer membrane proteins is due to their abundance in $\beta$-structure which is resistant to denaturation by SDS at low temperatures.

Recently, Laemmli's discontinuous buffer system ${ }^{5)}$ was used in slab SDS/PAGE which gave good separation of envelope proteins. ${ }^{\theta)}$ But the protein profiles differed entirely from those obtained in continuous buffer, and so the respondence of bands has not been established. In this note, we describe that some envelope proteins vary their migration rates depending on the buffer systems and we discuss their identity.

Envelope and cytoplasmic fractions were prepared from $E$. coli $\mathrm{K} 12$ YK 298 and solubilized at $37^{\circ} \mathrm{C}$ overnight as described previously. ${ }^{7)}$

In order to compare the mobilities of the proteins in SDS/PAGE with different buffer systems, we used twodimensional SDS/PAGE; the first dimension was SDS/ PAGE with continuous buffer system carried out in a glass tubing $(2.5 \mathrm{~mm}$ inside diameter) and the second dimension was SDS/PAGE with discontinuous buffer system carried out in a slab (0.8 mm thickness). Continuous buffer, used in the first dimension, was $0.1 \mathrm{M}$ sodium phosphate with $0.5 \% \operatorname{SDS}(\mathrm{pH} 7.1){ }^{8)}$ Dis-

Abbreviation: SDS/PAGE, SDS-polyacrylamide gel electrophoresis. continuous buffer system, used in the second dimension was as described by Laemmli: $:^{5)}$ The separation ge contained $0.375 \mathrm{M}$ Tris- $\mathrm{HCl}$ and $0.1 \%$ SDS (pH 8.8) the stacking gel, $0.125 \mathrm{M}$ Tris- $\mathrm{HCl}$ and $0.1 \%$ SDS (pF 6.8 ); and the electrode buffer, $0.025 \mathrm{M}$ Tris, $0.192 \mathrm{~N}$ glycine and $0.1 \%$ SDS (pH 8.3). Acrylamide con centrations were $7.5 \%$ and $10 \%$ for first and seconc dimensions, respectively. Gels were stained witt Coomassie brilliant blue R250 as described. ${ }^{\text {9) }}$ If the relative mobilities of the proteins are the same in firs 1 and second dimensions, proteins should be detected as spots on the diagonal line of the slab.

As shown in Fig. 1A, it was approximately the case for the cytoplasmic proteins of $E$. coli. Proteins smaller than M.W. ca. 15,000 were concentrated behind the dye front and observed as a line when $10 \%$ acrylamide was used in the second dimension SDS/PAGE (They can be separated if acrylamide concentration is increased). Flatness of each spot shows better separation of proteins in Laemmli's buffer system than in continuous one, which is a reason why extremely different protein profiles are obtained between the two buffer systems.

However, some deviations were observed when envelope proteins solubilized at $37^{\circ} \mathrm{C}$ overnight were analyzed (Fig. 1B). Several spots (spots a in Fig. 1B) deviated from the diagonal to the upper side, indicating that these proteins migrated faster in sodium phosphate buffer than in the Laemmli's discontinuous buffer system. Because most of them lined up vertically, they constituted a single band (band 13 of ref. 7) in continuous buffer, whereas they split into several bands in discontinuous one. This split could not be prevented by increasing concentration of SDS in Laemmli's buffer system up to $0.5 \%$, indicating that low concentration $(0.1 \%)$ of SDS in second dimension was not responsible for this split. These proteins were suggested to be outer membrane proteins because they were insoluble in sodium lauryl sarcosinate ${ }^{10)}$ (data not shown).

When envelope fraction was further solubilized at $100^{\circ} \mathrm{C}$ for $5 \mathrm{~min}$, all these deviating spots disappeared and three strong neighboring spots appeared on the diagonal line (spots $c, d$ and $e$ in Fig. 1C). As these spots lined up vertically, they constitute a strongest single band in continuous buffer system (see, for example, Fig. 1B of Ref. 2) whereas they split into three separate bands in Laemmli's buffer system. This shows excellent separation of proteins in Laemmli's buffer system.

When first dimension gel of unheated envelope fraction was boiled for $5 \mathrm{~min}$ in electrode buffer after electrophoresis and then subjected to second dimension SDS/PAGE, all these deviating proteins migrated downward as two strong neighboring spots below the diagonal (spots $f$ and $g$ in Fig. 1D) at the same horizontals as spots $\mathrm{c}$ and $\mathrm{d}$ in Fig. 1C. 

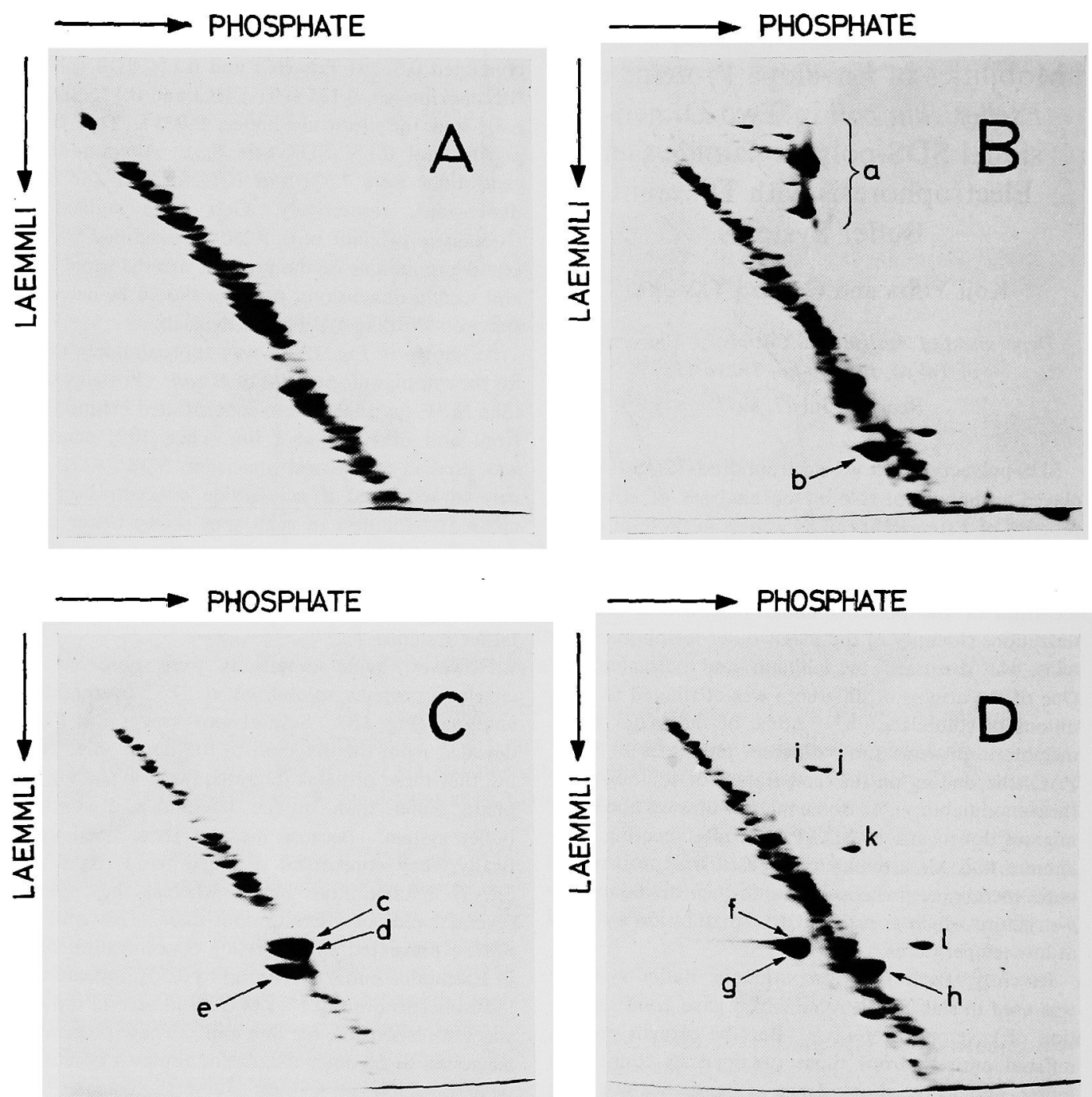

FIG. 1. Two-Dimensional SDS/PAGE Analyses of Proteins of E. coli K12 YK298 Grown in Nutrient Broth at $42^{\circ} \mathrm{C}$.

A, cytoplasmic proteins solubilized at $37^{\circ} \mathrm{C}$ overnight; $\mathrm{B}$, envelope proteins solubilized at $37^{\circ} \mathrm{C}$ overnight; $\mathrm{C}$, envelope proteins solubilized at $100^{\circ} \mathrm{C}$ for $5 \mathrm{~min}$; and $\mathrm{D}$, envelope proteins solubilized at $37^{\circ} \mathrm{C}$ overnight, and after SDS/PAGE in the first dimension they were boiled for $5 \mathrm{~min}$ and subjected to SDS/PAGE in the second dimension.

These results indicate that two downward heatmodifiable major outer membrane porteins, similar in molecular weight, split into several bands in Laemmli's buffer system before heating. These two proteins seem to be identical to $0-8$ and $0-9^{4)}$ because they were resistant to trypsin and pronase digestion and considerable part of them remained insoluble in $2 \%$ SDS at $60^{\circ} \mathrm{C}$ (data not shown). Though O-8 and O-9 were reported to bind strongly to peptidoglycan before heating, ${ }^{11)}$ similar amounts of them were detected on
Fig. 1C (spots $\mathrm{c}$ and $\mathrm{d}$ ) and Fig. 1D (spots $\mathrm{f}$ and $\mathrm{g}$ ). They seemed to be separated from peptidoglycan by electrophoresis (but not by centrifugation) and enter the gels, because electrostatic forces were thought to be important to their binding to peptidoglycan. ${ }^{\text {II) }}$

Similar observation was reported for major outer membrane proteins $0-8$ and $0-9$ by Nakamura and Mizushima ${ }^{4)}$ when continuous buffer system containing $8 \mathrm{M}$ urea was used. Deviating spots (spots a in Fig. 1B) were much reduced when first dimension gel con- 
tained $8 \mathrm{M}$ urea, suggesting that these proteins migrate similarly in Laemmli's buffer system and continuous buffer containing urea before heating. Nakamura and Mizushima ${ }^{4}$ showed that these major outer membrane proteins were rich in $\beta$-structure which was resistant to denaturation by SDS at low temperatures. These outer membrane proteins may present in several states which differ in terially structure and therefore they do not migrate to the right position in either first or second dimension SDS/PAGE before heating.

In addition to the results described above, following observations seem to be noticeable. Spot b in Fig. 1B is an upward heat-modifiable protein (band 18 of Ref. 7) and turned into spot e of Fig. 1C and spot h of Fig. 1D after heating; it is probably identical to $0-10.4$ In Fig. 1D, four new spots (i 1) absent in Fig. 1B appeared above the diagonal. These spots should represent upward heat-modifiable envelope proteins, which must be difficult to be identified in one-dimensional SDS/PAGE. Thus two-dimensional SDS/ PAGE should be useful in studying electrophoretic properties and identification of envelope proteins without purifying them.

Acknowledgment. This study was supported by a grant from the Ministry of Education, Science and
Culture of Japan.

\section{REFERENCES}

1) S. Ichihara and S. Mizushima, J. Biochem., 81, 1525 (1977).

2) M. Inouye and M. Yee, J. Bacteriol., 113, 304 (1973).

3) R. A. F. Reithmeier and P. D. Bragg, FEBS Lett., 41, 195 (1974).

4) K. Nakamura and S. Mizushima, J. Biochem., 80, 1411 (1976).

5) U. K. Laemmli, Nature, 227, 680 (1970).

6) G. F. Ames, J. Biol. Chem., 249, 634 (1974).

7) K. Yoda, K. Nagai, and G. Tamura, J. Biochem., 81, 1357 (1977).

8) M. Inouye and J. P. Guthrie, Proc. Natl. Acad. Sci. U.S. A., 64, 957 (1969).

9) G. Fairbanks, T. L. Steck and D. F. H. Wallach, Biochemistry, 10, 2606 (1971).

10) C. Filip, G. Fletcher J. L. Wulff and C. F. Earhart J. Bacteriol., 115, 717 (1973).

11) Y. Hasegawa, H. Yamada, and S. Mizushima, J. Biochem., 80, 1401 (1976). 